\title{
Análisis Estadístico De Los Residuos Sólidos Domésticos De La Parroquia San Sebastian Del Coca Del Cantón Joya De Los Sachas
}

\author{
Juan Pablo Wayllas Pazmiño \\ Magister en Gestión del Desarrollo Local Comunitario \\ Docente de Extensión Norte Amazónica ESPOCH, Ecuador \\ Luis Fabián Cabezas Arévalo \\ Ingeniero En Estadística Informática, Ecuador
}

\begin{abstract}
A statistical analysis was carried out on the characterization of domestic solid waste that was generated in the San Sebastian del Coca parish which belongs to the Canton Joya de los Sachas. This paper focuses on quantifying the generation of solid waste, calculating the per capita production, determining the composition and density of solid waste, and characterizing the amount and composition of the DSW generated by the samples taken from the San Sebastián del Coca parish. 48 families were chosen to carry out the research with an average of 4 inhabitants per family for a total of 192 people. As for the methodology, the simple random sampling method was used. A total of 96 dwellings samples that were obtained directly from the dwellings was chosen to be part of the study sample. The results showed that the generation of solid waste, in other words the PPC of domestic solid waste, was $0.87 \mathrm{~kg} / \mathrm{inhab} / \mathrm{day}$. This is with a total of $167.04 \mathrm{~kg} / \mathrm{day}$, which were collected by collector trucks and disposed according to the ordinance of the canton. The characterization of domestic solid waste in the parish has organic waste located in the first place with $78.2 \%$. Next was the generation of plastic bags with $6.19 \%$. This, therefore, was followed by cardboard with 5\%, plastic $7.7 \%$, and paper with $2.99 \%$.
\end{abstract}

Keywords: Analysis, Statistics, Waste, Solids, Sebastián del Coca, Orellana

Resumen

Se realizó un análisis estadístico sobre la caracterización de los residuos sólidos domésticos que se generaron en la parroquia San Sebastián del Coca del Cantón Joya de los Sachas. Los objetivos del estudio fueron cuantificar la generación de los desechos sólidos, calcular la producción per- 
cápita, determinar la composición y la densidad de los residuos sólidos y caracterizar la cantidad y composición de los RSD que generan las muestras tomadas de la Parroquia San Sebastián del Coca. Se escogieron 48 familias para realizar la investigación con un promedio de 4 habitantes por familia para un total de 192 personas. En lo que respecta a la metodología, se utilizó el método de muestreo aleatorio simple de un total de 96 viviendas, muestras que se obtuvieron directamente de las viviendas escogidas para ser parte de la muestra. Los resultados evidenciaron que la generación de residuos sólidos, es decir, el PPC de desechos sólidos domésticos, fue de $0,87 \mathrm{~kg} / \mathrm{hab} /$ día, con un total de $167,04 \mathrm{~kg} / \mathrm{dí} a$, que son recogidos por los carros colectores y eliminados de acuerdo a la ordenanza del cantón. La caracterización de los desechos sólidos domésticos en la parroquia ubica a los desechos orgánicos en primer lugar con $78,2 \%$, continúa la generación de fundas plásticas con el $6,19 \%$, seguido del cartón con el $5 \%$, plástico $7,7 \%$ y papel con el $2,99 \%$.

Palabras Clave: Development, Gongronema latifolium, Otuoke

\section{Introducción}

El manejo de los residuos sólidos constituye a nivel mundial un problema para las grandes ciudades. Factores como el crecimiento demográfico, la concentración de población en las zonas urbanas, el desarrollo ineficaz del sector industrial y/o empresarial, los cambios en patrones de consumo y las mejoras del nivel de vida, entre otros, han incrementado la generación de residuos sólidos en los pueblos y ciudades (Ojeda Lozano \& Quintero Whitty, 2008).

El manejo de estos residuos tiene también una estrecha relación con la salud de la población. En lo que a esto respecta, Se han presentado tres situaciones principales: la primera, referida a la transmisión de enfermedades por bacterias y parásitos tanto por agentes patógenos transferidos por los residuos como por vectores que se alimentan y reproducen en los residuos; en segundo lugar, el riesgo de lesiones e infecciones ocasionadas por los objetos punzo cortantes que se encuentran en los residuos, condición que pone en alto riesgo la salud de las personas que recuperan materiales en los vertederos; y en tercer lugar la contaminación ocasionada por la quema de residuos, la cual afecta el sistema respiratorio de los individuos (Contreras \& Maira, 2008).

El manejo de residuos sólidos está comprendido por todas las actividades funcionales u operativas relacionadas con la manipulación de los residuos sólidos desde el lugar donde son generados hasta la disposición final de los mismos (Ochoa Osvaldo, 2009). A continuación se describe la situación actual de las etapas que conforman este sistema para algunos países de América Latina y el Caribe. 


\section{Generación de residuos sólidos}

La generación constituye la primera etapa del manejo de residuos sólidos, y está directamente relacionada con las actividades que realiza el ser humano, el crecimiento poblacional, los cambios en los patrones de consumo, el incremento de la actividad industrial y comercial y las condiciones climáticas, entre otros factores (Ojeda Lozano \& Quintero Whitty, 2008).

En la Cumbre de la Tierra realizada en 1992 por la ONU en Río de Janeiro, se formularon cuatro áreas de programas relacionadas con los residuos: (a) reducción al mínimo de los residuos, (b) aumento al máximo de la reutilización y reciclado ecológico de los residuos, (c) promoción de la eliminación y el tratamiento ecológicamente racional de los residuos y (d) ampliación del alcance de los servicios que se ocupan de los desechos; esto con la finalidad de promover el desarrollo sostenible y ecológicamente racional para el siglo XXI en todos los países (ONU, 1992).

A nivel mundial, el incremento en el consumo de productos procesados ha elevado la tasa de generación de desechos diaria por habitante (Ochoa, 2009). En el caso latinoamericano, Ripoll (2003) indicó que la tasa de generación en las últimas décadas se ha incrementado de 0,5 a $1 \mathrm{Kg} /$ habitantedía, lo cual resulta inferior en un $25 \%$ a $50 \%$ a la tasa de generación de los países industrializados. Para 2005 la Organización Panamericana de la Salud (OPS) reportó que la tasa media per cápita de residuos sólidos urbanos asciende a 0,91 Kg/habitante-día para América Latina y El Caribe.

En el Ecuador, según los datos del INEC 2010, la cobertura de los servicios de recolección y disposición final de residuos sólidos a nivel nacional es de un promedio de $80,45 \%$. Los gobiernos locales se han visto limitados a enfrentar el problema debido a la mínima capacidad técnica de los responsables para la prestación de los servicios, la insuficiencia de recursos financieros, tasas y política tarifaria que no permiten cubrir los costos del servicio; la poca diversificación de los modelos de gestión y la falta de proyectos de minimización, recuperación, reciclaje y reaprovechamiento.

El inadecuado manejo de los residuos sólidos se evidencia en el deterioro del ambiente, el cual sufre una considerable alteración en la calidad de los recursos como: agua, suelo y aire, lo que conlleva a la alteración del ecosistema.

El impacto global de los residuos sólidos está creciendo rápidamente. Los desechos sólidos son una gran fuente de generación de gas metano, un importante generador de gases de efecto invernadero con gran impacto a corto plazo.

Un manejo inadecuado de los residuos sólidos contribuye a las inundaciones, contaminación del aire y los impactos en la salud pública (enfermedades respiratorias, diarrea, dengue entre otras). El impacto ambiental generado por la inadecuada disposición de residuos sólidos se 
evidencia tanto en la contaminación de cuerpos hídricos superficiales y subterráneos como de los ecosistemas en general (PNGIDS).

\section{Composición de residuos sólidos}

La información referente a la cantidad de residuos sólidos generados por una región y la caracterización de los mismos constituye una gran herramienta para la planificación del proceso de recolección de los mismos y el diseño de los sistemas de eliminación a utilizar (Sharholy et al., 2008).

Los porcentajes de materiales reciclables como cartón y papel son relativamente bajos. Esto puede ser ocasionado por el nivel socioeconómico de los pobladores, sus patrones de consumo y el hecho de que en muchos de esos países los materiales reciclables son recuperados por sectores informales en las distintas etapas funcionales del manejo de residuos sólidos (Organización Panamericana de la Salud \& Organización Mundial de la Salud, 2005).

En el Ecuador, según datos suministrados por el Programa Nacional de Gestión Integral de Desechos Sólidos, el MIDUVI y otras instituciones, se determinó que el servicio de recolección de residuos sólidos tiene una cobertura nacional promedio del $84.2 \%$ en las áreas urbanas y de $54.1 \%$ en el área rural. La fracción no recolectada contribuye directamente a la creación de micro basurales descontrolados.

Apenas un 24\% de los Gobiernos Autónomos Descentralizados ha iniciado procesos de separación en la fuente, $26 \%$ procesos de recuperación de materia orgánica y $32 \%$ de recolección diferenciada de desechos hospitalarios. El $73,4 \%$ de los vehículos de recolección del país son compactadores y se tiende a no utilizar equipos abiertos. El 70\% de los equipos supera la vida útil de 10 años.

Sólo el $28 \%$ de los residuos son dispuestos en rellenos sanitarios, sitios inicialmente controlados que con el tiempo y por falta de estabilidad administrativa y financiera, por lo general, terminan convirtiéndose en botaderos a cielo abierto. El $72 \%$ restante de los residuos es dispuesto en botaderos a cielo abierto (quebradas, ríos, terrenos baldíos, etc.), que provocan inconvenientes e impactos de diferente índole como taponamiento de cauces de agua y alcantarillados, generación de deslaves, proliferación de insectos y roedores, los cuales traen consigo problemas ambientales y de salud a la población.

Actualmente, la generación de residuos en el país es de 4,06 millones de toneladas métricas al año y una generación per cápita de $0,74 \mathrm{~kg}$. Se estima que para el año 2017 el país generará 5,4 millones de toneladas métricas anuales, por lo que se requiere de un manejo integral planificado de los residuos. 
Las acciones de control y seguimiento emprendidas por el Ministerio del Ambiente se han basado en los artículos 46 y 125 del Libro VI referente a Calidad Ambiental, estipulados en el Texto Unificado de Legislación Secundaria de Medio Ambiente (TULSMA), y las acciones actuales en las políticas ambientales nacionales relacionadas con el manejo de desechos que el Ministerio del Ambiente emitió mediante Acuerdo Ministerial $\mathrm{N}^{\circ}$ 86, del 11 de noviembre del 2009.

Aunque la Legislación Ambiental vigente ha considerado en el Libro VI, Anexo VI del TULMAS Normas de Calidad Ambiental para el manejo y disposición final de Desechos Sólidos no peligrosos, en pro de contribuir con el medio ambiente y reconocer el derecho de la población a vivir en un ambiente sano y ecológicamente equilibrado que garantice la sostenibilidad del buen vivir, se han realizado algunos alcances a la legislación, como la publicación del Acuerdo Ministerial 031, en la que se incluyen los procesos de cierre técnico y saneamiento de botaderos de los desechos sólidos y viabilidad técnica. De igual manera se incluye el tratamiento de desechos peligrosos en los Acuerdos Ministeriales 026, 161 y 142 y de desechos especiales en el Acuerdo 190 (Política Nacional de Post-consumo de equipos eléctricos y electrónicos).

El Gobierno Nacional, a través del Ministerio del Ambiente, crea en abril del año 2010 el PROGRAMA NACIONAL PARA LA GESTIÓN INTEGRAL DE DESECHOS SÓLIDOS (PNGIDS), con el objetivo primordial de impulsar la gestión de los residuos sólidos en los municipios del Ecuador con un enfoque integral y sostenible y con la finalidad de disminuir la contaminación ambiental, mejorando la calidad de vida de los ciudadanos e impulsando la conservación de los ecosistemas a través de estrategias, planes y actividades de capacitación, sensibilización y estímulo a los diferentes actores relacionados.

El programa ha iniciado una nueva etapa para eliminar los botaderos a cielo abierto de todos los municipios del país hasta diciembre de 2017.

En la provincia de Orellana la gestión de los desechos sólidos se realiza de acuerdo a la Ordenanza única ambiental $\mathrm{N}^{\mathbf{0}}$ 303, Orellana-Ecuador., GADMFO., 2012., Pp. 113-121, del Gobierno Autónomo Descentralizado Municipio de Francisco de Orellana (Solvesa, 2017).

En la Parroquia San Sebastián habitan personas de diferentes partes del Ecuador, es por eso que cada familia tiene sus propias costumbres, gastronomía, religión entre otras.

Ninguna familia hace el debido reciclaje de la basura,; algunos sólo lo hacen de las botellas plásticas porque existe una remuneración económica. El recolector de basura circula dos veces a la semana (miércoles y domingos), razón por la cual los habitantes deben recolectar la basura en recipientes con un volumen considerable (tanques, saquillos, etc.) para evitar la acumulación 
de residuos que representa una contaminación visual al hogar y a la parroquia en general, disminuyendo así la posibilidad de contraer enfermedades por roedores, mosquitos y animales domésticos que pueden destruir los recipientes en los que las familias del sector recolectan la basura.

\section{Materiales Y Métodos}

\section{Parroquia San Sebastián del Coca}

Ubicación: La parroquia de San Sebastián del Coca es parte del cantón Joya de los Sachas, en la provincia de Orellana, se ubica a $16 \mathrm{~km}$ de la Cabecera del Cantonal Joya de los Sachas.

Límites: al Norte limita con la provincia de Sucumbíos; al Sur con las comunidades San José y Amaru Mesa del cantón Francisco de Orellana; al Este con la Parroquia de Rumipamba, Tres de Noviembre, Lago San Pedro, Joya de los Sachas y San Carlos, al Oeste con Río Coca.

Altitud: Oscila entre 380 y 260 metros sobre el nivel del mar.

Clima: Su clima es húmedo y tropical, nubosidad media es de 5.5 octas. Su temperatura media es de $25.5^{\circ} \mathrm{C}$.

Superficie: Con una superficie de $283.4 \mathrm{Km} 2$.

Gráfico $\mathbf{N}^{\circ} 1$ Parroquia San Sebastián del Coca

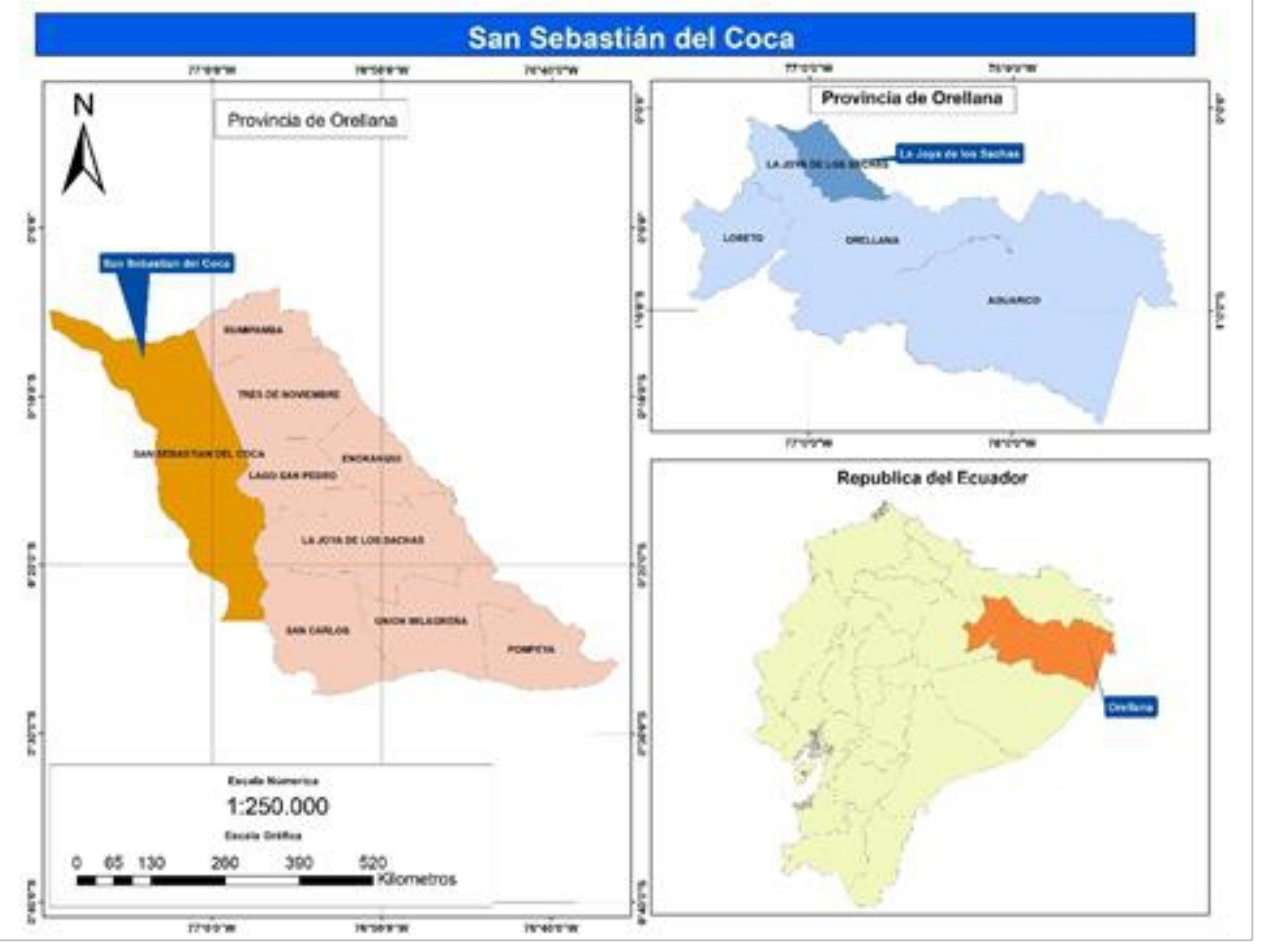

Fuente: GAD Parroquial San Sebastián del Coca 


\section{Población}

La población de la Parroquia San Sebastián del Coca cuenta con 96 casas y 384 habitantes.

\section{Área de estudio}

La ejecución de este proyecto se realizó en la Parroquia San Sebastián del Coca, Cantón Joya de los Sachas, la cual está ubicada en la parte centro norte de la ciudad de Francisco de Orellana, área donde se tomaron los datos y muestras de los residuos sólidos domésticos.

La toma de datos geográficos se realizó mediante la utilización de un GPS que ayuda a ubicar un punto en el espacio. Estos datos se pueden procesar posteriormente sea manualmente o mediante uso de un software especializado y obtener los planos correspondientes del área de estudio.

\section{Muestreo de residuos sólidos} Muestreo Aleatorio Simple

Consistió en escoger de las unidades muéstrales un total de 48 viviendas, de tal modo que cada una tenga la misma posibilidad de ser escogida.

\section{Verificación y Actualización de la Información Urbana}

Consistió en la búsqueda de información específica, estadística y actualizada, de datos que servirán como base del estudio:

\section{Población}

Se realizaron encuestas, donde se obtuvieron los datos del número exacto de la población, en este caso para las 96 viviendas de la Parroquia San Sebastián del Coca.

\section{Estructura de las Encuestas}

- Datos para determinar el número de personas que viven en cada domicilio, tipo de vivienda, pisos.

- Servicios Básicos: Barrido y Recolección de los residuos sólidos, tipo de transporte que utiliza, telefonía e internet.

- Utilización de recipientes.

- Frecuencia de recolección en ese sector.

- Disponibilidad para ayudarnos con este proyecto.

\section{Estudio de caracterización}

Se utilizó la siguiente metodología para determinar las características de los desechos sólidos de la parroquia: 
- Se solicitó la colaboración del GAD Parroquial San Sebastián del Coca.

- Se realizó una reunión de socialización con los moradores que fueron seleccionados para el muestreo.

- Se identificaron las viviendas objeto del muestreo.

- Las muestras se recolectaron directamente de las viviendas.

\section{Producción per cápita}

Para establecer la producción per cápita de desechos sólidos en la parroquia San Sebastián del Coca se realizó la toma de muestras por un lapso de 21 días consecutivos. Se procedió a tomar el peso de los desechos para establecer su volumen. A fin de determinar la producción per cápita se utilizó la fórmula:

$$
\begin{gathered}
\text { PPC }=\frac{\text { peso de la basura }(\mathrm{kg} / \text { día }}{\text { número de habitantes (hab) }} \\
\text { PPC }=\frac{167,04}{192} \\
\text { PPC }=0,87
\end{gathered}
$$

Para establecer la caracterización de los desechos sólidos de la parroquia San Sebastián del Coca se procedió a recolectar los desechos sólidos directamente de los domicilios, trasladados a un centro de acopio donde se pesaron y clasificaron los mismos.

\section{Población y muestra Población}

La parroquia San Sebastián del Coca cuenta con 96 viviendas, según el censo poblacional del 2010, con un promedio de 4 habitantes por vivienda, arrojando un total de 384 personas.

\section{Muestra}

Para determinar el número de muestras para la Parroquia San Sebastián del Coca se utilizó la siguiente fórmula.

$$
\mathrm{n}=\frac{\mathrm{z}^{2} \mathrm{pqN}}{\mathrm{e}^{2}(\mathrm{~N}-1)+\mathrm{z}^{2} \mathrm{pq}}
$$

Dónde:

N= Tamaño conocido de población (96)

$\mathrm{e}=$ Error máximo permitido $(10 \%)$

$\mathrm{Z}=$ Margen de confiabilidad (1.96) 
$\mathrm{p}=$ Probabilidad de que el evento ocurra $(0.5)$

$\mathrm{q}=$ Probabilidad de que el evento ocurra $(0.5)$

$\mathrm{n}=$ Tamaño de la muestra

$$
\begin{gathered}
n=\frac{(1.96)^{2}(0.5)(0.5)(96)}{(0.1)^{2}(96-1)+(1.96)^{2}(0.5)(0.5)} \\
n=\frac{92,1984}{1,9104} \\
n=48.2 \\
n=48
\end{gathered}
$$

\section{Resultados}

De las 48 familias escogidas para realizar el estudio, con un promedio de 4 habitantes por cada una de ellas que da un total de 192 habitantes, los resultados se evidencian en la tabla N. 2

Tabla N.1. Producción per cápita:

\begin{tabular}{|l|c|}
\hline NÚMERO DE FAMILIAS & 92 \\
\hline PROMEDIO DE HABITANTES POR FAMILIA & 5,13 \\
\hline PROMEDIO DE PRODUCCIÓN DE RESIDUOS & 5,79 \\
\hline
\end{tabular}

Figura N. 1. Estratos de viviendas

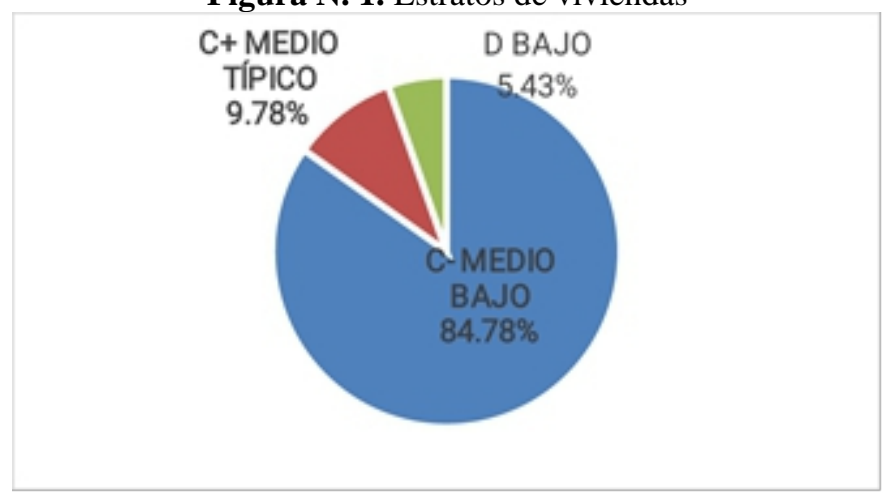

En la parroquia San Sebastián del Coca la PPC de la provincia de Orellana fue de $0,87 \mathrm{~kg} / \mathrm{hab} /$ día, arrojando una producción diaria de 167 $\mathrm{kg} / \mathrm{día}$.

\section{Caracterización de los desechos sólidos}

En lo que se refiere a la caracterización de los residuos sólidos, los resultados se evidencian en la tabla N. 2. 
Tabla N. 2. Caracterización de desechos sólidos

\begin{tabular}{|c|c|c|c|c|}
\hline \multirow{2}{*}{ SUBPRODUCTOS } & \multicolumn{2}{|c|}{ Sector 1} & \multicolumn{2}{c|}{ Sector 2} \\
\cline { 2 - 5 } & $\begin{array}{c}\text { Promedio en } \\
\mathrm{Kg}\end{array}$ & $\%$ & $\begin{array}{c}\text { Promedio en } \\
\mathrm{Kg}\end{array}$ & $\%$ \\
\hline ORGÁNICO & 42,84 & $86,7 \%$ & 40,31 & $73,0 \%$ \\
\hline PLÁSTICO & 2,04 & $4,1 \%$ & 7,43 & $13,5 \%$ \\
\hline VIDRIO & 1,78 & $3,6 \%$ & 1,98 & $3,6 \%$ \\
\hline PAPEL Y CARTÓN & 1,32 & $2,7 \%$ & 2,53 & $4,6 \%$ \\
\hline TEXTIL & 0,43 & $0,9 \%$ & 1,50 & $2,7 \%$ \\
\hline LATAS & 0,99 & $2,0 \%$ & 1,45 & $2,6 \%$ \\
\hline TOTAL & $\mathbf{4 9 , 4 1}$ & $\mathbf{1 0 0 , 0 \%}$ & 55,19 & $100,0 \%$ \\
\hline
\end{tabular}

Una vez que se han pesado los desechos orgánicos, se evidencia que los de mayor peso fueron los residuos domésticos, seguidos de fundas plásticas, cartón, plástico y papel.

Una vez clasificados los desechos por productos, se pesan por separado. Para obtener el peso en porcentaje de cada uno de ellos se utiliza la siguiente ecuación:

$$
\mathrm{P} \%=\frac{\mathrm{G} 1}{\mathrm{G}} * 100
$$

Dónde:

$\mathrm{P} \%=$ porcentaje del subproducto

$\mathrm{G} 1=$ peso del subproducto

$\mathrm{G}=$ peso total de los subproductos

\section{Discusión}

Según la Secretaría Nacional de Planificación y Desarrollo, en la Gestión Integral de Desechos Sólidos en el artículo 55 literal d) de la COOTAD, es responsabilidad de los municipios el manejo de los desechos sólidos. A nivel nacional se determina un bajo nivel de gestión en las municipalidades en dicha responsabilidad; esto se demuestra en los datos referentes a la disposición final de los residuos sólidos que indican que el $80 \%$ lo realizan en botaderos y $20 \%$ en rellenos sanitarios (Fuente: MAE-PNGIDS 2013). A su momento, la mayoría de los municipios cerraron las unidades para proveer el servicio bajo la dependencia jerárquica de las direcciones de higiene; en otros, se hizo a través de las comisarías municipales. En resumen, La capacidad de gestión del el manejo de los residuos sólidos a nivel municipal no es la más eficiente debido a que por lo general estas entidades municipales no cuentan con autonomía administrativo-financiera.

En el Ecuador se generaron aproximadamente 4'139.512 Tm de residuos, con un promedio diario de generación per cápita promedio de 0,73 $\mathrm{kg} / \mathrm{hab}$-día a nivel nacional. 
Según el Programa 'PNGIDS' del Ministerio del Ambiente del Ecuador de los 221 Gobiernos Autónomos Descentralizados Municipales (GADM), al año 2014 el 65\% dispone sus residuos en botaderos a cielo abierto, quebradas y orillas de cuerpos de agua, mientras que el 35\% dispone sus residuos en rellenos sanitarios manuales, mecanizados y mancomunados. En el Ecuador, según datos del año 2013, se generan alrededor de 11.341 toneladas diarias de residuo; es decir, un aproximado de 4'139.512 Tm/año.

Con respecto a la caracterización de los residuos sólidos en el país, el $61,4 \%$ son orgánicos, papel + cartón $9.4 \%$, plástico $11 \%$, vidrio $2.6 \%$, chatarra $2.2 \%$, y otros $13.3 \%$. De los 221 Gobiernos Autónomos Descentralizados Municipales (GADM), hasta el año 2014 el 65\% dispone sus residuos en botaderos a cielo abierto, quebradas y orillas de cuerpos de agua, mientras que el 35\% dispone sus residuos en rellenos sanitarios manuales, mecanizados y mancomunados.

En la revista European Scientific Journal January 2018 edition Vol.14, No.2 ISSN: 1857 - 7881 (Print) e - ISSN 1857- 7431 se publica el artículo Estudio Estadístico de la Caracterización de desechos sólidos en la parroquia Taracoa de la provincia de Orellana. En éste la PPC fue de 0,89 kg/hab-día, generando un total de 273,60 kg/día. La PPC de la parroquia San Sebastián del Coca fue de $0,87 \mathrm{~kg} / \mathrm{hab} /$ día, lo cual confirma la veracidad de los resultados alcanzados.

\section{Conclusiones}

En la parroquia San Sebastián del Coca la generación de residuos sólidos no es adecuada pues no se realiza el reciclaje de los desechos sólidos. Se pudo observar que se reciclan las botellas plásticas únicamente, debido a que los carros recolectores de basura acuden al sector dos veces por semana (miércoles y domingo). La basura es colocada en saquillos o tanques muy grandes, lo cual genera la presencia de insectos, roedores y animales domésticos que destruyen los sacos donde se encuentra la basura regándola por toda la calle; esto puede producir enfermedades y contaminación del medio ambiente.

La producción per cápita de desechos sólidos domésticos en la parroquia San Sebastián del Coca fue de 0,87 kg/hab/día, arrojando una producción diaria de 167 kg/día.

Realizado el análisis estadístico sobre la caracterización de los desechos sólidos domésticos en la parroquia en primer lugar se ubican los desechos orgánicos con 78,2\%, continúa la generación de fundas plásticas con el $6,19 \%$, seguido del cartón con el $5 \%$, plástico $7,7 \%$ y papel con el 2,99 $\%$. 


\section{References:}

1. Contreras, S. \& Maira, J. (2008). Evaluación de experiencias locales urbanas desde el concepto de sostenibilidad: el caso de los desechos sólidos del municipio de Los Patios (Norte de Santander, Colombia).Trabajo Social. 109-134.

2. Ochoa Osvaldo (2009). Recolección y disposición final de los desechos sólidos, zona metropolitana. Caso: Ciudad Bolívar.

3. Ojeda Lozano \& Quintero Whitty (2008). Generación de residuos sólidos domiciliarios por periodo estacional: el caso de una ciudad mexica-na. I Simposio Iberoamericano de Ingeniería de Residuos. Castellón.

4. ONU (1992). Conferencia mundial sobre el medio ambiente y el desarrollo: :http://www.un.org/esa/dsd/dsd/dsd_faqs_csd.shtml\#Q5. Recuperado el 20 de Septiembre de 2012.

5. Organización Panamericana de la Salud \& Organización Mundial de la Salud (2005). División de Salud y Ambiente. Informe de la evaluación regional de los servicios de manejo de residuos sólidos municipales en América Latina y el Caribe. Washington, DC.

6. http://www.ambiente.gob.ec/programa-pngids-ecuador/ Programa Nacional para la Gestión Integral de Desechos Sólidos - PNGIDS ECUADOR.

7. PNGIDS. Programa Nacional para la Gestión Integral de Desechos Sólidos - PNGIDS ECUADOR [Internet]. Available from: http://www.ambiente.gob.ec/programa-pngids-ecuador.

8. Sharholy, M., Ahmad, K., Mahmood, G., \& Trivedi, R.C. (2008). Municipal solid waste management in India cities- A review. Waste Management, 28, 459-467.

9. Solvesa (2017). Plan de manejo de desechos sólidos en la Gestión Ambiental [Internet]. [cited Nov 12]. Available from: http://www.solvesacorp.com/solvesacorp.com/docs/downloads/Plan de manejo de desechos sólidos en la Gestión Ambiental.pdf. 